\title{
Chemical Imaging on the Nanoscale - Top-Illumination Tip-Enhanced Raman Spectroscopy
}

\author{
Johannes Stadler*, Thomas Schmid, and Renato Zenobi \\ §SCS Metrohm Prize for best oral presentation
}

\begin{abstract}
A top illumination system for tip-enhanced Raman spectroscopy (TERS) in a gap-mode configuration is presented here, which allows chemical analysis of sample surfaces with a lateral resolution beyond the optical diffraction limit and optical detection of very small amounts of analyte molecules (down to single molecule sensitivity). The technique combines the high resolution of an STM with label-free, chemical-rich information of Raman spectra at ambient pressure. In this system, using a special geometry with illumination and detection perpendicular to the sample surface, a lateral resolution of $<15 \mathrm{~nm}$ was achieved using low laser powers and split second acquisition times per spectrum. This was achieved due to a very high enhancement of the Raman signals in the order of $10^{7}$ by etched metal tips, and allowed the acquisition of $64 \times 64$ to $200 \times 200$ pixels chemical Raman maps with full spectral information in every pixel within a reasonable time frame (<25 min for $64 \times 64$ pixels). The Raman maps give simultaneous information about localization and chemical nature of a sample with high sensitivity and high resolution.
\end{abstract}

Keywords: Gap-mode TERS · Nanoscale chemical analysis · Scanning tunneling microscopy · Spectroscopic imaging · Tip-enhanced Raman spectroscopy

\section{Introduction}

In the past few years, advances in miniaturization have spread from research into development and industrial production and continued further on in research. One concern for continually shrinking structures and components is a lack of control due to missing techniques for high-resolution chemical characterization. Information about topography and structures can be easily acquired using atomic force microscopy (AFM), scanning tunneling microscopy (STM) as well as scanning/transmission electron microscopy (SEM/TEM). Disadvantages of these techniques are limited chemical information and a need for performing the measurement in high vacuum (in the case of electron microscopy (EM)).

\footnotetext{
${ }^{\star}$ Correspondence: J. Stadler

ETH Zurich

Department of Chemistry and Applied Biosciences

Wolfgang-Pauli-Strasse 10, HCI D 325

$\mathrm{CH}-8093$ Zurich

Tel.: +4144632 4377

Fax: +41446321292

E-Mail: stadler@org.chem.ethz.ch
}

A higher content of chemical information is available from vibrational spectroscopy techniques such as infrared (IR) and Raman spectroscopy (RS). They are, however, limited to around $\lambda / 2$ in lateral resolution by the diffraction limit of light (with $\lambda$ denoting the wavelength of the light). Raman spectroscopy is limited by the intrinsically low scattering cross section.

Tip-enhanced Raman spectroscopy (TERS), a combination of AFM or STM with RS was first described in 2000. ${ }^{[1]}$ It combines the strengths of both techniques - chemical information with high lateral resolution. As opposed to other techniques this is achieved at ambient pressure. In TERS, a fully metallic STM[2] or metallized AFM tip ${ }^{[1 \mathrm{a}, 3]}$ is approached to the sample surface of interest (inside the optical near-field) and centered in the laser focus of a confocal Raman microscope. Several processes then enhance and confine the signal of the molecules on the surface. Collective oscillations of electrons in the metal (surface plasmons) channel the collected energy from the laser beam and confine it to a strongly localized electromagnetic field (EM) at the apex of the tip which interacts with the molecule to emit Raman signals. These signals are again amplified by the metal tip and scattered back into the far field for detection. Simultaneously, the signal of the sample is restricted to the extent of the EM field, i.e. to the area directly underneath the apex of the tip, increasing the spatial resolution of the technique to the order of tens of nanometers. Essentially TERS is creating a very small and very strong 'nano-torch' which can be used as an excitation light source for optical experiments.

Several different geometries for TERS have been realized, which can be grouped into two main categories. In bottom illumination TERS, ${ }^{[1 \mathrm{a}, 2,4]}$ the excitation laser light is introduced from underneath the sample using a refraction index matched high numerical aperture (NA) objective. This requires transparent samples but yields a very well confined confocal spot size and high local EM fields. Side illumination TERS ${ }^{[5]}$ is realized by a long working distance objective under an angle of $30-50^{\circ}$ sacrificing focusing and collection efficiency to gain distance to the sample, but increasing the amount of possible samples by removing the restriction for sample transparency. A lesser used geometry is top illumination which allows the use of higher NA objectives and a symmetric illumination reducing the confocal spot size. Two approaches have been presented until now. Meixner and Pettinger have successfully implemented a parabolic mirror (PM) as focusing element ${ }^{[6]}$ which allows for very high NA at the sacrifice of easy handling of the system (as the PM has very high requirements towards the optical alignment 


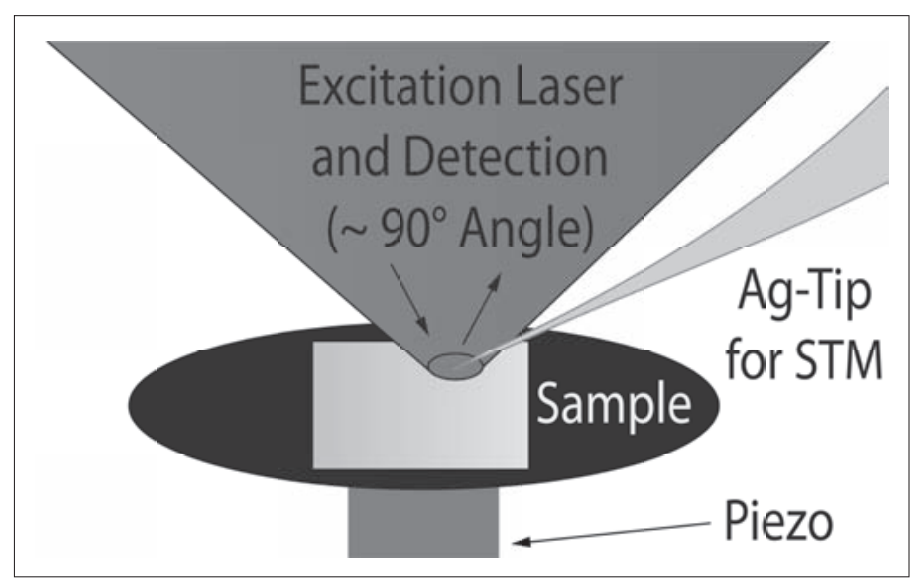

Fig. 1. Schematic representation of the TERS setup used in this study. Angled Ag tip in STM feedback in close proximity to the sample surface centered in a focused laser beam. Sample fixed on a $100 \times 100 \times 10$ um scanning piezo. $632.8 \mathrm{~nm} \mathrm{He}-\mathrm{Ne}$ or 532 nm solid-state laser light is delivered and collected by a lens in top-illumination (Lens: Mitutoyo, NA 0.7).

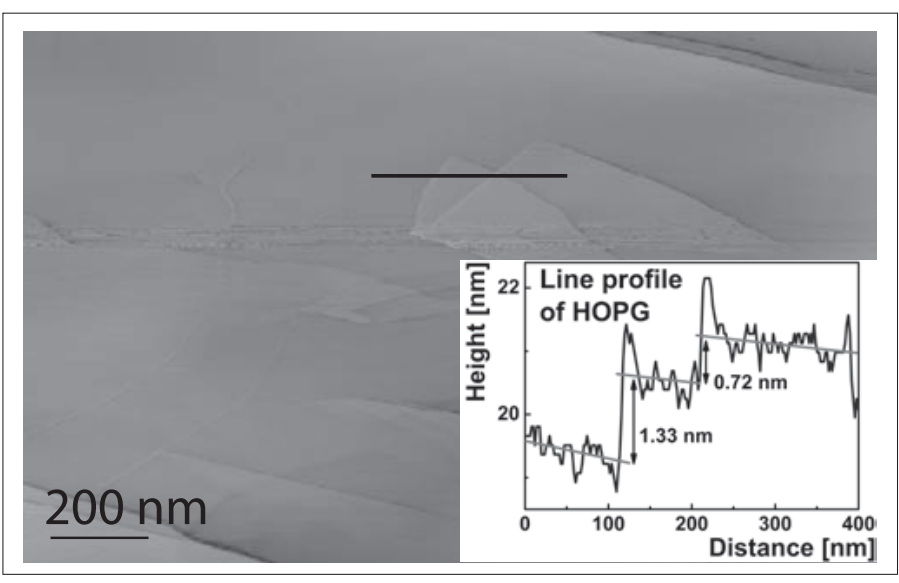

Fig. 2. STM scan of a flat highly ordered pyrolytic graphite (HOPG) surface with an etched silver tip, $2 \mathrm{~nm}$ step size. The image shows steps of graphite sheets on the HOPG surface with $0.72 \mathrm{~nm}$ and 1.33 $\mathrm{nm}$ height respectively, corresponding to 2 and 4 layers of graphite. Inset: height profile of the graphite sheets. Noise level $<0.5 \mathrm{~nm}$, lateral resolution of $<15 \mathrm{~nm}$ during scan (linear correction applied). of the system $\left.{ }^{[7]}\right)$. The second approach was shown by Schultz et al. based on a standard lens for focusing, but yielding contrast between near-field and far-field signal of 2 and a resolution of 200-300 $\mathrm{nm},{ }^{[8]}$ maybe limited due to the small NA of the lens they used. The limited signal-to-noise ratio only allowed them to determine their enhanced signal after background correction for the confocal far-field contribution.

We present an intermediate approach with a NA 0.7 lens, which allows both a reasonably comfortable handling of the lens and the confined field of a high NA focusing element. This lens focuses a standard Gaussian mode laser beam onto the end of an etched silver STM tip in gap mode configuration.

'Gap mode' TERS describes a situation in which the dipole induced into the metal STM tip is mirrored by a close-by metallic surface ${ }^{[9]}$ creating a very strong and confined electromagnetic (EM) field in between the induced dipole in the tip and its mirror image in the metal surface. We can benefit from this strong localized field and the additional field enhancement due to the lightning-rod effect ${ }^{[10]}$ and a suitable surface plasmon resonance ${ }^{[11]}$ of the etched silver tips in close proximity to gold sample substrates. As depicted in Fig. 1, the etched silver tip is introduced at an angle of $30-50^{\circ}$ with respect to the sample surface.

In this study we demonstrate TERS imaging with high pixel numbers and a lateral resolution of $<15 \mathrm{~nm}$ revealing the distribution of organic dye molecules on gold surfaces.

\section{Experimental}

The experiments were conducted on an adapted commercial STM/Raman instru- ment (NT-MDT, Russia, NTEGRA Spectra Upright) equipped with a $100 \times 100 \times 10$ $\mu \mathrm{m}$ piezo scanning stage, a long working distance objective for excitation and collection of optical signals (Mitutoyo, 100x $\mathrm{NA}=0.7$ ), as well as a quadruple grating spectrometer with an EMCCD camera (Andor, Newton 971) for detection (a more detailed description of the instrument can be reviewed in ref. [12]).

Solvents were purchased from various suppliers (incl. Aldrich, Fluka, Acros) in analytical grade purity. As analytes, brilliant cresyl blue (BCB, Fluka) and Nile blue A (NB, Fluka) dyes, dissolved and diluted in methanol were used.

Freshly cleaved pieces of mica (ProSciTech, Australia, V2) were coated with 100 nm of gold (Bal-Tec, 99,99\%) by resistive heating, using a deposition rate around 0.1 $\mathrm{nm} / \mathrm{s}$ at pressures below $10^{-5}$ mbar. The coated mica was used as conductive sample support for STM. The structure of the gold coating was determined by AFM and STM and showed corrugation of less than $5 \mathrm{~nm}$ over the entire, otherwise flat surface.

The substrates were spin-coated with $10 \mu \mathrm{L}$ of different BCB or NB solutions. Coated metal films were controlled confocally to exclude surface-enhanced Raman scattering (SERS) activity.

The STM tips were electrochemically etched at $10 \mathrm{~V}$ from $0.25 \mathrm{~mm}$ silver wire using a $1 \mathrm{~cm}$ gold loop as counter electrode and an etchant solution of 1:1-1:2 (v/v) perchloric acid (Riedel de Haën) : methanol. The current cutoff was controlled by a custom-made device ${ }^{[13]}$ to stop etching upon formation of a sharp tip. The tip shape was controlled visually under a $360 \times$ stereomicroscope for correct etching before use and tips with a diameter of $75 \mathrm{~nm}$ and less could be routinely produced. ${ }^{[12]}$ Selected tips combined suf- ficient sharpness with sufficient stiffness for STM scanning.

\section{Results and Discussion}

\section{STM Properties}

Fig. 2 shows an STM image from a test measurement with an etched STM tip on a clean HOPG surface at $2 \mathrm{~nm} /$ pixel resolution demonstrating the scan performance of the instrument. In the image, edges of single HOPG layers can be clearly resolved. The line profile in the inset determines the step height in between layers as $0.72 \mathrm{~nm}$ and $1.33 \mathrm{~nm}$, corresponding to 2 and 4 layers of freshly exfoliated graphene (expected height of a single step is 0.335 $\left.\mathrm{nm}^{[14]}\right)$. The average noise level in the measurement can be estimated as $0.5 \mathrm{~nm}$ and a lateral resolution of less than $10 \mathrm{~nm}$ was achieved. Consecutive scans showed a thermal drift of $2.2 \mathrm{~nm} / \mathrm{min}$ over $2 \mathrm{~h}$, not accounting for differences in sample properties, sample fixation or changes in room temperature.

\section{Full Spectroscopic TERS Imaging}

Recording a TERS map involves several steps, starting with an alignment of tip and focused laser beam. For this, the tip is ideally located on a homogeneous sample and raster scanned with the focused laser beam, monitoring the Raman intensity as in Fig. 3(a) (a Raman spectrum is recorded in every pixel and the intensity of a marker band is evaluated). The generated Raman map allows a very precise alignment to the strongest enhancing site on the tip and is a great improvement over previous alignments guided by external microscopes ${ }^{[5 b, 15]}$ (which can potentially miss a close-by stronger TERS site). The image in Fig. 3(a) includes in- 


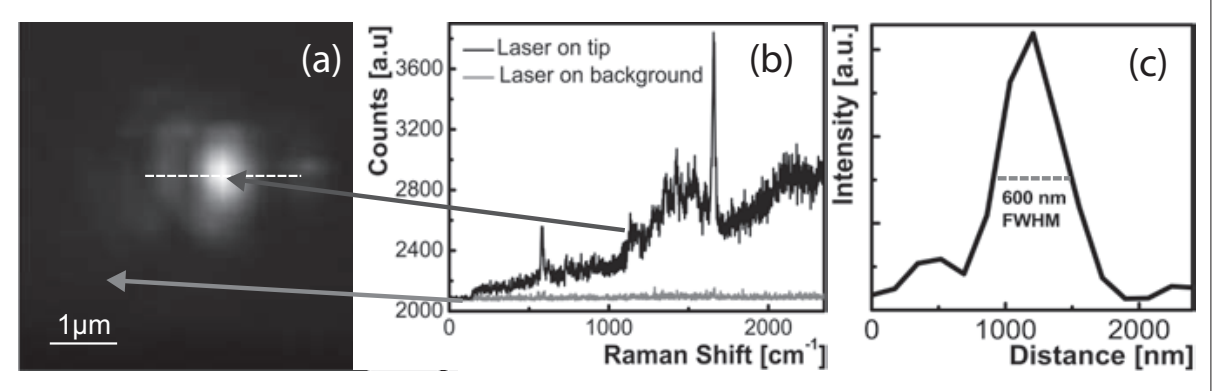

Fig. 3. (a) $5 \times 5 \mu \mathrm{m}$ image representing the BCB intensity of the area around the tip on a sample of BCB $10 \mu \mathrm{L} 5 \times 10^{-5} \mathrm{~mol} / \mathrm{L}$ on gold obtained by rastering the laser over the approached tip (tip entering from the right hand side). Arrows indicate pixels from which the spectra in (b) were taken. (b) TERS spectrum acquired on the tip (grey), Raman spectrum obtained from unenhanced background (black) showing a contrast of $>20$; 1 s per spectrum. (c) Line profile over the enhanced area in (a). The confocal focus size can be deduced from the intensity profile along the dotted line to be around $600 \mathrm{~nm}$.
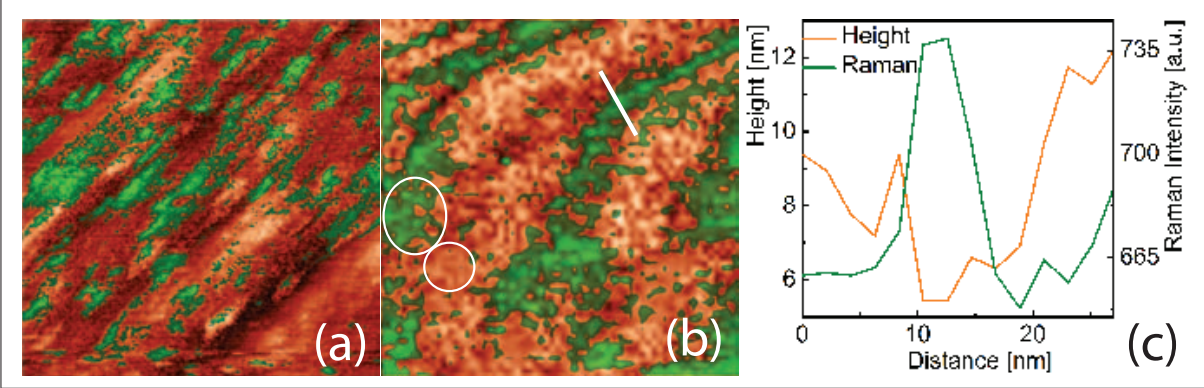

Fig. 4. TERS maps of a (a) $400 \times 400 \mathrm{~nm}$ and a (b) $128 \times 128 \mathrm{~nm}$ area of a spin-coated sample of $10 \mu \mathrm{L} 5 \times 10^{-4} \mathrm{~mol} / \mathrm{L}$ NB on gold, $200 \times 200$ and $64 \times 64$ pixels respectively, $0.05 \mathrm{~s}$ per pixel. Images show the overlay of Raman intensity at $580-600 \mathrm{~cm}^{-1}$ (green, translucent) on top of the topography signal (orange/red, total height in the topography images is 14 and $16 \mathrm{~nm}$ respectively). (c) Height and intensity profile along the white line shown in (b) indicating higher Raman intensity in a trench localized to a width of $<15 \mathrm{~nm}$. White circles show areas of high and low Raman intensity without obvious topographic difference, ruling out tip-sample distance fluctuations as origin of the intensity changes.

formation about the spectral background intensity as well as the enhanced signals on the tip (shown in Fig. 3(b) in the black/ grey spectrum). As additional information from this map, the confocal focus size of the excitation laser can be determined by a line cut over the slightly elongated, circular shaped enhancement region (as there are stronger signals whenever the intensity from the laser focus overlaps with the enhancing tip). In our case the focus size is around $600 \mathrm{~nm}$ in diameter (Fig. 3(c)). The laser is then aligned to the strongest enhancing location (indicated by the upper arrow) and interlocked. All movements (laterally as well as tip-sample distance control) from this point on are carried out by the piezo stage of the instrument to keep the relative positions of tip and laser stable and maintain the enhancement.

For the actual measurement of interest, the sample-scanning piezo stage rasters the sample underneath the tip, a Raman spectrum is collected in every pixel and stored as a 4D matrix ( $\mathrm{x}, \mathrm{y}$, Raman shift and intensity). From this matrix, each spectrum can be reviewed separately or intensity distributions for specific Raman marker bands (specific for a certain molecular species) can be extracted.

During the recording of the spectra, additional information (topography, STM feedback error signals and the Rayleigh scattering intensity) is obtained and can be combined for evaluation as in Fig. 4(a) and (b). There, a combination of topography and Raman intensity displays the chemical composition (from the marker bands) with high lateral resolution. Fig. 4(a) and (b) are two Raman maps of a 45 nm-thick gold film, spin-coated with $10 \mu \mathrm{L}$ of $5 \times$ $10^{-4} \mathrm{~mol} / \mathrm{L}$ Nile blue. Both images show the topographic signal from the STM feedback in orange/red superimposed with the Raman intensity of the NB marker band at $590 \mathrm{~cm}^{-1}$ in green. Both spectra have a step size of $2 \mathrm{~nm} /$ pixel and a lateral size of (a) $400 \times 400 \mathrm{~nm}$ and (b) $128 \times 128 \mathrm{~nm}$ respectively. In both measurements, the increased Raman intensity forms coherent patterns within several lines and thus noise or random distance fluctuations can be excluded as origin for these signals. In a confocal scan, the entire image would be inside a single confocal diameter of the laser beam, and thus form a single homogeneous pixel - but by increasing the resolution using TERS, higher intensity of NB inside small 2-3 nm deep trenches in the gold film can be visualized. The enhanced signal strength is strongly depending on the tip-sample distance, ${ }^{[16]}$ but the fact that even flat areas in the white circles in Fig. 4(b) exhibit strong spectral differences indicates that TERS can detect changes in concentration on the surface (maybe even down to the single molecule level as shown in other publications $\left.{ }^{[1 \mathrm{a}, 15 \mathrm{~b}, 16 \mathrm{~b}]}\right)$.

\section{High Resolution Chemical Imaging}

The line profile for the STM height and the Raman intensity in Fig. 4(c) are extracted from the same location (white line) in Fig. 4(b) and clearly show the correlation between the highest Raman intensity and the trenches in the gold film (most likely, dye molecules accumulate in the trenches or at the boundaries). From these line cuts, the resolution of the STM and TERS experiment can be deduced to be well below $15 \mathrm{~nm}$ laterally. In comparison to the size of the STM tip ( $r=75 \mathrm{~nm})$, the field is confined to a fraction of the tip apex only, clearly confirming its near-field origin (similar observations have been made experimentally by Hartschuh et al. ${ }^{[17]}$ as well as calculations by Roth et al. ${ }^{[11 b]}$ support this).

\section{High Pixel Number Raman Maps}

Another key criterion for imaging is the time requirement for an image and, related to that, the time per spectrum. The overall acquisition time for a single experiment is limited by the stability of the system (tiplaser alignment, sample drift, feedback range etc.). Due to the highly enhanced signals in these TERS experiments, the time/spectrum in Fig 4(a) and (b) could be reduced down to $0.05 \mathrm{~s}$ allowing for higher pixel numbers within a reasonable time per image. The overall image time for Fig. 4(a) and (b) was less than $2 \mathrm{~h}$ and 10 minutes, respectively. Previous experiments were limited in pixel number, due to far longer collection times necessary to yield the spectral information and partly required tedious manual subsequent acquisition of spectra with manual repositioning of the tip ( $>6 \mathrm{~h}$ for $20 \times 20$ pixels, ${ }^{[18]}>10 \mathrm{~min}$ for $16 \times 16$ pixels, ${ }^{[19]}>22 \mathrm{~h}$ for $64 \times 64$ pixels $\left.{ }^{[20]}\right)$. The faster the scanning, the higher number of pixels that can be recorded, allowing the user to choose between either a higher lateral resolution or a scan of larger sample area.

A further advantage of a highly enhancing tip is the opportunity to reduce the laser power to as few as $15 \mu \mathrm{W}$ (Fig. 
4). This is possible due to 'hot' tips and the better confinement of the light onto the tip apex, aiding in conserving fragile samples by reducing thermal strain on the sample. Using variable laser power, similar intense spectra could be produced with laser powers as low as $4 \mu \mathrm{W}$, albeit at longer integration times, showing that a choice between acquisition time and spatial resolution and laser power can now be made. This increases the flexibility to account for different sample properties and gain a maximum of information (fast imaging with high power for stable inorganic samples, or low power measurements to prevent delicate biological samples from overheating and disintegrating).

\section{Enhancement Factor}

The enhancement factor (EF) in TERS reflects how many Raman photons each molecule contributes to the overall Raman signal in a TERS experiment in comparison to a confocal Raman experiment. As explained before, this determines how fast or with how much laser power one can measure.

$$
E F=\frac{I_{N F}}{I_{F F}} \cdot \frac{A_{F F}}{A_{N F}}
$$

A lower boundary for the achieved enhancement factor EF (Eqn. (1)) can be calculated by taking into consideration the intensity of the far field ( $\mathrm{I}_{\mathrm{FF}}$, tip retracted) and near-field Raman signals ( $\mathrm{I}_{\mathrm{NF}}$, tip approached) and by normalizing them to the area (and with that to the number of molecules) from which they originate in the far-field $\left(\mathrm{A}_{\mathrm{FF}}\right)$ and near-field $\left(\mathrm{A}_{\mathrm{NF}}\right)$ cases.

The diameter of the confocal laser spot size $\left(\mathrm{A}_{\mathrm{FF}}\right)$ has been determined in Fig. 2(c) as $600 \mathrm{~nm}$. The diameter of the near-field spot size $A_{\mathrm{NF}}$ was determined from the smallest resolved features in Fig. 4(c) to be $15 \mathrm{~nm}$.

For the intensity, the amount of signal in the $590 \mathrm{~cm}^{-1}$ Raman marker band, corrected by the surrounding background was chosen. In Fig. 5 spectra extracted from a Raman map of a sample of Nile blue dye (NB) on a flat gold film are shown. The far field intensity $\mathrm{I}_{\mathrm{FF}}$ from the background is shown in Fig. 5 and equals 350 counts in $50 \mathrm{~s}$. In the near field case $\left(\mathrm{I}_{\mathrm{NF}}\right)$, the strongest Raman signals (usually chosen for evaluation) were up to 21000 counts in $0.05 \mathrm{~s}$. However we consider it more correct to average over the entire Raman map (roughly corresponding to the same size as the confocal focus), amounting to 2000 counts in $0.05 \mathrm{~s}$. Spectra 1 and 2 in Fig. 5 were chosen arbitrarily from a Raman map and show that the spectra within this TERS map were very similar (indicating a clean sample without contaminations), but differ

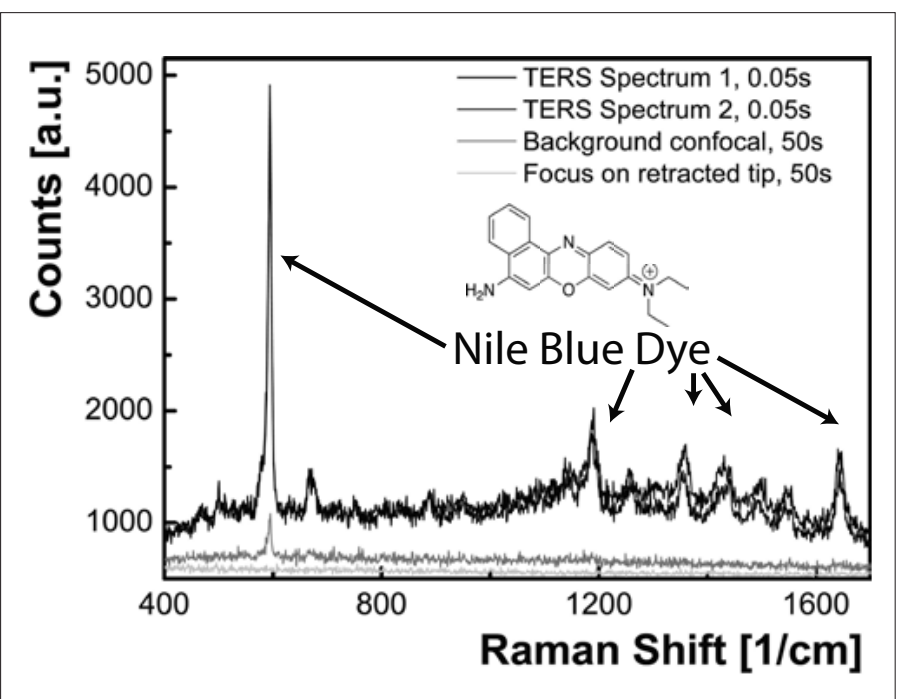

Fig. 5. Raman spectra from a Raman map of NB on a flat gold surface; (black) 2 random TERS spectra, 0.05 s showing the spectral reproducibility within the map; (dark grey) 50 s confocal Raman spectrum (tip retracted); (light grey) 50 s spectrum from the tip end (tip retracted, after measurement) to check cleanliness of tip. Inset: chemical structure of used Nile blue dye.

slightly in enhancement or in the number of probed molecules.

With these values, the enhancement can then be calculated to $9 \times 10^{6}$ over the entire TERS map, or to $>10^{8}$ for the strongest spectra.

No high pixel number, full spectral maps have been presented in literature. Previous Raman maps either were of much lower pixel number ${ }^{[19,21,22]}$ or were from selected spectral bands only. ${ }^{[8,23]}$ This work presents a very important step for TERS towards exact, high-resolution localization of substances and detection of concentration differences. The geometry with illumination from the top offers a series of advantages over presently used geometries, and can compete with or even outperform them. The enhancement of $\sim 10^{7}$ is in line with the highest enhancement factors reported lately from other groups which range from $10^{5}$ to $10^{7},[21,24]$ even though they may determine enhancement factors in different ways. A standardized way of determining the EF has already been proposed in, ${ }^{[25]}$ and could lead to better comparability between publications.

The spatial resolution of $15 \mathrm{~nm}$ has also been reached in other publications, [21,22b,26] and is amongst the best in the field.

\section{Conclusions}

In conclusion, a top-illumination, gapmode TERS geometry with a lateral resolution of $<15 \mathrm{~nm}$ has been presented. The system can gather this highly localized chemical information either with very low laser powers or in split second acquisition times per spectrum, due to the high en- hancement of $>10^{7}$. These studies present a potential of TERS in additional applications, i.e. controlling the production processes of small structures like molecular electronics or inorganic patterns or delivering information about molecular selfassembly or maybe even about catalysis on metal surfaces.

\section{Acknowledgements}

The authors thank I. Olliges-Stadler for SEM measurements and the Roche Research Foundation for funding.

Received: January 10, 2011

[1] a) R. M. Stoeckle, Y. D. Suh, V. Deckert, R. Zenobi, Chem. Phys. Lett. 2000, 318, 131; b) M. S. Anderson, Appl. Phys. Lett. 2000, 76, 3130 ; c) N. Hayazawa, Y. Inouye, Z. Sekkat, S. Kawata, Optics Commun. 2000, 183, 333. d) B. Pettinger, G. Picardi, R. Schuster, G. Ertl, Electrochem. 2000, 68, 942

[2] B. Pettinger, G. Picardi, R. Schuster, G. Ertl, Single Mol. 2002, 3, 285.

[3] N. Hayazawa, Y. Inouye, Z. Sekkat, S. Kawata Chem. Phys. Lett. 2001, 335, 369.

[4] B. Pettinger, G. Picardi, R. Schuster, G. Ertl, J. Electroanal. Chem. 2003, 554, 293.

[5] a) W. H. Zhang, T. Schmid, B. S. Yeo, R. Zenobi, Isr. J. Chem. 2007, 47, 177; b) B. Pettinger, B. Ren, G. Picardi, R. Schuster, G. Ertl, J. Raman Spec. 2005, 36, 541; c) D. Mehtani, N. Lee, R. D. Hartschuh, A. Kisliuk, M. D. Foster, A. P. Sokolov, J. F. Maguire, J. Raman Spec. 2005, 36, 1068; d) N. Hayazawa, M. Motohashi, Y. Saito, H. Ishitobi, A. Ono, T. Ichimura, P. Verma, S. Kawata, J. Raman Spec. 2007, 38, 684.

[6] a) M. Sackrow, C. Stanciu, M. A. Lieb, A. J Meixner, Chem. Phys. Chem. 2008, 9, 316; b) J. Steidtner, B. Pettinger, Rev. Sci. Instrum. 2007, 78, 103104; c) C. Stanciu, M. Sackrow, A. J. Meixner, J. Microscopy - Oxford 2008, 229, 247.

[7] A. Drechsler, M. Lieb, C. Debus, A. Meixner, G. Tarrach, Opt. Express 2001, 9, 637. 
[8] Z. D. Schultz, S. J. Stranick, I. W. Levin, Appl. Spec. 2008, 62, 1173.

[9] P. I. Geshev, K. Dickmann, J. Optics A Pure App. Opt. 2006, 8, S161.

[10] L. Novotny, R. X. Bian, X. S. Xie, Phys. Rev. Lett. 1997, 79, 645.

[11] a) A. L. Demming, F. Festy, D. Richards, J. Chem. Phys. 2005, 122, 7; b) R. M. Roth, N. C. Panoiu, M. M. Adams, R. M. Osgood, C. C. Neacsu, M. B. Raschke, Opt. Express 2006, 14 2921.

[12] J. Stadler, T. Schmid, R. Zenobi, Nano Lett. 2010, 10, 4514

[13] a) J. P. Ibe, J. P. P. Bey, S. L. Brandow, R. A Brizzolara, N. A. Burnham, D. P. DiLella, K. P. Lee, C. R. K. Marrian, R. J. Colton, J. Vac. Sci. Technol. A: Vac. Surf. Films 1990, 8, 3570; b) W. H. Zhang, X. D. Cui, B. S. Yeo, T. Schmid, C. Hafner, R. Zenobi, Nano Lett. 2007, 7, 1401.

[14] G. Bacon, Acta Cryst. 1951, 4, 558.

[15] a) W. H. Zhang, B. S. Yeo, T. Schmid, R. Zenobi, J. Phys. Chem. C 2007, 111, 1733; b) Y. Saito, M. Motohashi, N. Hayazawa, M. Iyoki, S. Kawata, Appl. Phys. Lett. 2006, 88, 143109.

[16] a) M. B. Raschke, C. Lienau, Appl. Phys. Lett. 2003, 83, 5089; b) C. C. Neacsu, J. Dreyer N. Behr, M. B. Raschke, Phys. Rev. B 2006 , 73, 4; c) K. F. Domke, 'Tip-enhanced Raman Spectroscopy - Topographic and Chemical Information on the Nanoscale', Freie Universitä Berlin (Berlin), 2006; d) B. Pettinger, K. F. Domke, D. Zhang, R. Schuster, G. Ertl, Phys. Rev. B 2007, 76, 113409 .
[17] A. Hartschuh, E. J. Sanchez, X. S. Xie, L. Novotny, Phys. Rev. Lett. 2003, 90, 4.

[18] V. Deckert, D. Zeisel, R. Zenobi, T. Vo-Dinh, Anal. Chem. 1998, 70, 2646.

[19] D. Zhang, X. Wang, K. Braun, H. J. Egelhaaf, M. Fleischer, L. Hennemann, H. Hintz, C. Stanciu, C. J. Brabec, D. P. Kern, A. J. Meixner, J. Raman Spec. 2009, 40, 1371.

[20] Y. Saito, P. Verma, K. Masui, Y. Inouye, S. Kawata, J. Raman Spec. 2009, 40, 1434

[21] J. Steidtner, B. Pettinger, Phys. Rev. Lett. 2008, 100, 236101.

[22] a) G. Picardi, M. Chaigneau, R. Ossikovski, C. Licitra, G. Delapierre, J. Raman Spec. 2009, 40, 1407; b) A. Hartschuh, H. Qian, C. Georgi, M. Bohmler, L. Novotny, Anal. Bioanal. Chem. 2009, 394, 1787; c) X. Wang, D. Zhang, K. Braun, H. J. Egelhaaf, C. J. Brabec, A. J. Meixner, Adv. Funct. Mater. 2010, 20, 492.

[23] a) Y. Saito, M. Motohashi, N. Hayazawa, S. Kawata, J. Microscopy - Oxford 2008, 229, 217 ; b) G. G. Hoffmann, G. de With, J. Loos, Macromol. Symp. 2008, 265, 1.

[24] Z. Liu, X. Wang, K. Dai, S. Jin, Z.-C. Zeng, M.-D. Zhuang, Z.-L. Yang, D.-Y. Wu, B. Ren, Z.-Q. Tian, J. Raman Spect. 2009, 40, 1400.

[25] T. Schmid, B. S. Yeo, W. Zhang, R. Zenobi, in 'Tip Enhancement', Eds. S. Kawata, M. V. Shalaev, Elsevier, Amsterdam, 2007, pp. 115155.

[26] N. Anderson, A. Hartschuh, L. Novotny, Nano Lett. 2007, 7, 577. 